\title{
Edge Detection Method of Iris Image
}

\author{
Xianglong Zhou ${ }^{1}$, Jingbo Zhao ${ }^{2}$, kuoting wang ${ }^{1}$ \\ ${ }^{1}$ Navy Submarine Academy, Qingdao, Shandong Province, China \\ ${ }^{2}$ Qingdao Technological University, Qingdao, Shandong Province, China \\ zx1159@sina.com, zhaojingbo6666@163.com
}

\begin{abstract}
The paper describes several edge detection operators, such as Roberts operator, Prewitt operator, Sobel operator, Laplace operator and Canny operator. They were used to detect the edge of iris image. The better one among them for detecting edge of iris image was chosen.
\end{abstract}

Index Terms - iris image, edge detection, iris identification

\section{Introduction}

In iris identification systems, the localization of boundary of interior edge and outer edge ${ }^{[1]}$ of iris images is the first step of the process. The edge of images is a set of pixels around which the variation of grey level of pixels are of pulse type or roof type, Poggio defined edge detection as " measurement, detection and boundary localization of grey changes of image mainly "[2]. Edge plays a great role in image division, pattern recognition and machine vision etc. Several edge detection operators, such as Roberts operator, Prewitt operator, Sobel operator, Laplace operator and Canny operator are introduced and used to detect the image of iris in the paper. Finally, a better one of these operators for detecting the edge of iris image was selected.

\section{Digital Image Edge Detection}

The edge detection of digital image is an important foundation in image division, recognition of object and shapes pick-up. Poggio said: "The edge either corresponds to the boundary of object or not, but the edge has satisfied properties, it can greatly reduce the information which should be dealt with as well as it keeps the shape information of object in image." The edge of object appears some characteristic of the image. For example, the luminance of pixels on edge changes remarkably, such as sudden change of grey level, sudden change of color and sudden change of structure etc. Actually, the edge of object is the boundary of different regions. There are two properties for edge of image: direction and magnitude. Usually, the change of grey level along the edge is mild and the change of grey level perpendicular to the edge is acute. According to the characteristic of grey level, three kinds of edge could be defined: the first is step-edge (fig.1, a) where the difference of grey level between both side of edge is quite big; the second is roof-edge (fig. 1 b). The grey level of image from one side to another side increases firstly, then it reduces slowly. The third is line-edge (fig. $1 \mathrm{c}$ ), the grey levels of image jumps from one to anther, then back to original. Of course, the edge of image in practical situation is more complicated, and the number of kind of grey variation along edge is not limited to the above mentioned.

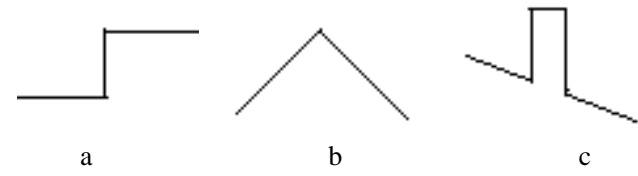

Fig. 1 line-edge

\section{The Operators for Edge Detection}

The edge detection of object is important in image processing and image recognition. The edge of object always appears in the variation of grey level of image. Sometimes, the object could be identified by using coarse edge only. There are several kinds of detection operators for edge detection:

The first one is the differential operator. Obviously, the place with relative large variation of grey level in image is corresponding to the place with relatively large value of gradient function. The differential operator is a better way to do it. It is well known that there are several operators commonly used, such as Roberts operator, Prewitt operator and Sobel operator.

The second one is Laplace operator. Because the places where the gradients reach maximum values (positive or minus) are the edge. Therefore, they are the inflexion points of grey function of image. From the view of mathematics, 2-order derivative of the function at flex point is 0 .

The third is Canny operator. Canny operator utilizes the 1-order derivative of Gauss function and can make better compromise between noise rejection and edge detection.

\section{A. Differential operators}

The edge detection could be done by convolution of differential operator. In fact, the differential in digital image could be approximated by difference. Differential operator can be used not only in 2-D edge detection, but also in 3-D edge detection.

The sharp degree of the edge is determined by grey level of image. The most important property of the gradient of image is the gradient direction along which the variation of grey level is the biggest. Therefore, the gradients reflect the grey level variations in the edge of image exactly.

For image $f(x, y)$, the definition of gradient in $(x, y)$ is

$$
\operatorname{grad}(x, y)=\left[\begin{array}{l}
f_{x}^{\prime} \\
f_{y}^{\prime}
\end{array}\right]=\left[\begin{array}{l}
\frac{\partial f(x, y)}{\partial x} \\
\frac{\partial f(x, y)}{\partial y}
\end{array}\right]
$$


The magnitude and direction of gradient is

$$
\begin{aligned}
& \operatorname{grad}(x, y)=\sqrt{f_{x}^{\prime 2}+f_{y}^{\prime 2}}=\sqrt{\left(\frac{\partial f(x, y)}{\partial x}\right)^{2}+\left(\frac{\partial f(x, y)}{\partial y}\right)^{2}} \\
& \theta=\arctan \left(f_{y}^{\prime} / f_{x}^{\prime}\right)=\arctan \left(\frac{\partial f(x, y)}{\partial y} / \frac{\partial f(x, y)}{\partial x}\right)
\end{aligned}
$$

For digital image, difference replaces derivative and 1 -order difference is

$$
\begin{aligned}
& \Delta_{x} f(i, j)=f(i, j)-f(i-1, j) \\
& \Delta_{y} f(i, j)=f(i, j)-f(i, j-1)
\end{aligned}
$$

The difference of direction is

$$
\Delta_{\theta} f(i, j)=\Delta_{x} f(i, j) \cos \theta+\Delta_{y} f(i, j) \sin \theta
$$

The direction along which the direction derivative of the function $f(x, y)$ becomes the maximum is

$$
\theta=\arctan \left(f_{y}^{\prime} / f_{x}^{\prime}\right)
$$

The maximum value of the direction derivative is

$$
G[f(x, y)]=\left[\left(\frac{\partial f}{\partial x}\right)^{2}+\left(\frac{\partial f}{\partial y}\right)^{2}\right]^{\frac{1}{2}}
$$

It is called gradient template. The gradient operator is a better way to detect the edge because it has some useful properties, such as displacement invariability and Isotropic. To simplify the computation of gradient, an approximate form of gradient template could be adopted as follows

$$
\operatorname{grad}(x, y)=\max \left(\left|f_{x}^{\prime}\right|,\left|f_{y}^{\prime}\right|\right)
$$

or

$$
\operatorname{grad}(x, y)=\left|f_{x}^{\prime}\right|+\left|f_{y}^{\prime}\right|
$$

The operators used are Roberts operator, Prewitt operator and Sobel operator. The template of Roberts is shown in Fig.2
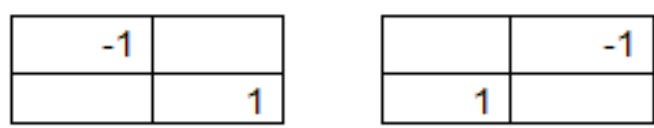

Fig. 2 Roberts template

The differences are

$$
\begin{aligned}
& f_{x}^{\prime}=|f(x+1, y+1)-f(x, y)| \\
& f_{y}^{\prime}=|f(x+1, y)-f(x, y+1)|
\end{aligned}
$$

The advantage of Roberts operator is accurate in detecting the edge of image, but it is sensitive to noise. Roberts operator is suitable for the detecting the image with clear edge and weaker noise.

For the sake of reducing the influence of noise on edge detection, Prewitt operator strengthened the template of operator such that the template is modified from $2 \times 2$ to $3 \times 3$ shown in Fig. 3.

\begin{tabular}{|l|l|l|}
\hline-1 & -1 & -1 \\
\hline 0 & 0 & 0 \\
\hline 1 & 1 & 1 \\
\hline
\end{tabular}

\begin{tabular}{|l|l|l|}
\hline-1 & 0 & 1 \\
\hline-1 & 0 & 1 \\
\hline-1 & 0 & 1 \\
\hline
\end{tabular}

Fig. 3 Prewitt template

Prewitt operator suppresses the noise by the averaging image information, but the average of image information is equivalent to a low-pass filter. Therefore, the boundary localization by Prewitt operator is not good as Roberts operator.

Based on Prewitt operator, Sobel operator computes the difference with weights in 4-domain as shown in Fig. 4.

\begin{tabular}{|l|l|l|}
\hline-1 & 0 & 1 \\
\hline-2 & 0 & 2 \\
\hline-1 & 0 & 1 \\
\hline
\end{tabular}

\begin{tabular}{|l|l|l|}
\hline-1 & -2 & -1 \\
\hline 0 & 0 & 0 \\
\hline 1 & 2 & 1 \\
\hline
\end{tabular}

Fig. 4 Sobel template

Sobel operator and Prewitt operator both are average operators with weight, but Sobel operator believe that the influence of the neighboring pixels to the present pixel is not equivalent. It means that the neighboring pixels with different distance have different weight and have different influence on the present pixel. Generally speaking, the further the distance is, the less the influence is.

\section{B. Laplace operator}

Laplace operator is a method of edge detection by using 2-order differential. It detects the edge points by searching the crossing zero point of 2-order differential of grey value. The detecting principal is as follows: the edge with slowly varying grey level is corresponding to an unimodal function after differential operation; the position of the peak value of the unimodal function corresponds to the edge point. The differential at the peak value of the unimodal function is zero and the signs at both sides of edge are opposite; Therefore, the peak point of unimodal function corresponds to the crossing zero point of 2-order differential function. The edge of image could be detected by the crossing zero point of 2-order differential function.

The Laplace expression is

$$
\nabla^{2} f=\frac{\partial^{2} f}{\partial x^{2}}+\frac{\partial^{2} f}{\partial y^{2}}
$$

For the discrete function $f(i, j)$, the Laplace expression is $\nabla^{2} f(i, j)=\Delta_{x}^{2} f(i, j)+\Delta_{y}^{2} f(i, j)$ where $\Delta^{2}{ }_{x} f(i, j)$ and $\Delta^{2} f(i, j)$ are 2-order differentials of 
image grey value in directions of $x$ and $y$. The expression of Laplace operator of discrete function is

$$
\begin{aligned}
\nabla^{2} f(i, j)= & f(i+1, j)+f(i-1, j)+f(i, j+1) \\
& +f(i, j-1)-4 f(i, j)
\end{aligned}
$$

The template of Laplace operator commonly used is shown in Fig. 5. The basic requirments of Laplacian template are: The coefficient at the central pixel is positive; The coefficients at the neighboring pixels are negative and the sum of coefficients for all pixels is zero.

\begin{tabular}{|c|c|c|}
\hline 0 & -1 & 0 \\
\hline-1 & 4 & -1 \\
\hline 0 & -1 & 0 \\
\hline
\end{tabular}

Fig.5 Laplacian template

As the Laplace operator is a 2-order differential, it is sensitive to noise. In addition, the Laplace operator usually produces the edge with double width and can not offer the information about edge direction. Therefore, Laplace operator is used hardly to detect the edge directly and is used mainly in deciding which side of the edge the pixels locate after detecting the edge of image.

\section{Canny operator}

In recent years, Canny operator has been got extensive application in image processing. On the basis of validity of edge detection and reliability of boundary localization, Canny studied the characteristic in optimizing edge detector and derived the mathematic expression to optimize edge detector. Canny pointed out three indexes to evaluate the performance for detecting image edge:

1) Good ratio of signal-to-noise, i.e., the probability of mistaking the non-edge point as the edge point should be small, vice versa.

2) Good performance of boundary localization, i.e., the edge points detected should be at the center of actual edge as possible.

3)The response of each edge point is exclusive, i.e., the edge detected should be the one with width of one point. Canny firstly expressed the above definition in mathematical form and adopted the optimizing numerical method to get the optimizing template of edge detection corresponding to the given type of edge.

Suppose the Gauss function as

$$
G(x, y)=\frac{1}{2 \pi \sigma^{2}} \exp \left(-\frac{x^{2}+y^{2}}{2 \sigma^{2}}\right)
$$

In direction $n$, the 1-order directional derivative of $G(x, y)$ is

$$
G_{n}=\frac{\partial G}{\partial n}=n \nabla G
$$

where

$$
n=\left[\begin{array}{l}
\cos \theta \\
\sin \theta
\end{array}\right] \quad \nabla G=\left[\begin{array}{l}
\partial G / \partial x \\
\partial G / \partial y
\end{array}\right]
$$

$\mathrm{n}$ is the directional vector and $\nabla G$ is the gradient vector. As changing the direction of $n$, the convolution of $f(x, y)$ with $G_{n}$, i.e., $G_{n} * f(x, y)$, reaches maximum, i.e.,

$$
\begin{aligned}
& \partial\left(G_{n} * f(x, y)\right) / \partial n=0 \\
& \frac{\partial\left(G_{n} * f(x, y)\right)}{\partial n}=\frac{\partial\left[\left(\cos \theta \bullet \frac{\partial G}{\partial x}\right) * f(x, y)+\left(\sin \theta \bullet \frac{\partial G}{\partial y}\right) * f(x, y)\right]}{\partial \theta}=0
\end{aligned}
$$

By then, the direction of $\mathrm{n}$ is perpendicular to the

direction of edge. The direction

$$
n=\frac{\nabla G * f(x, y)}{|\nabla G * f(x, y)|}
$$

In the direction $\mathrm{n}, G_{n} * f(x, y)$ gets the maximal output response.

$$
\begin{aligned}
\left|G_{n} * I\right| & =|\cos \theta(\partial G / \partial x) * f(x, y)+\sin \theta(\partial G / \partial y) * f(x, y)| \\
& =\left|\nabla G^{*} f(x, y)\right|
\end{aligned}
$$

Canny operator is a newer edge detection operator. It has good performance to detect edge and is used extensively. The edge detection of Canny utilizes 1-order differential and it can make compromise between noise suppression and edge detection.

\section{Application of Edge Detection Operator to Iris Identification}

After describing some edge detection operators commonly used, they have been tested in detecting the edge of iris. The testing results are shown in Fig.6 Fig. 10. From the results as shown, it could be seen clearly that the edges detected by Prewitt operator, Sobel operator and Canny operator are better, the edges detected by Roberts operator and Laplace operator are confuse. Fig. 7, Fig. 8 and Fig. 10 shown that Prewitt operator can suppress noise, but it loses some useful information. On the contrary, Canny operator keeps too much useless information and the computation is too complicate to implement. In iris identification system, the edge detection is the basis of boundary localization of circle. Therefore, the Sobel operator was the better one due to better result, simplicity and easy to program.

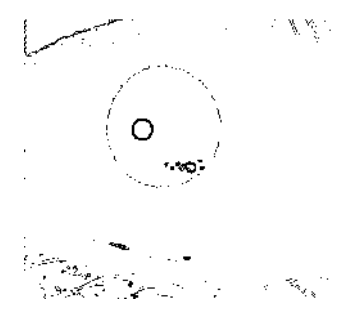

Fig. 6 Roberts operator

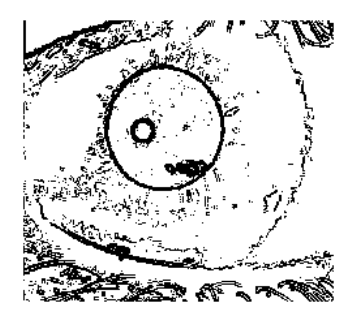

Fig. 7 Prewitt operator 


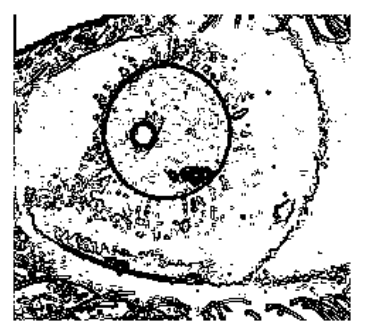

Fig. 8 Sobel operator

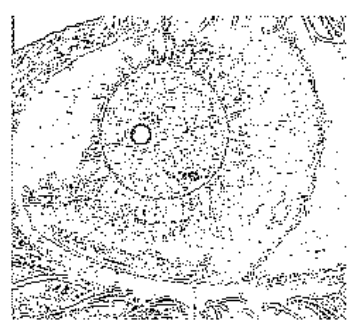

Fig. 9 Sobel operator

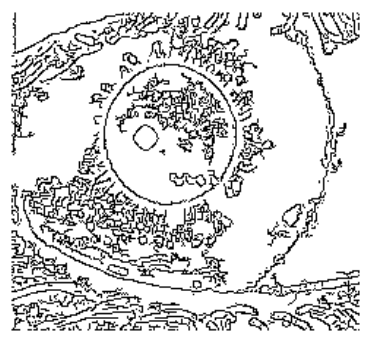

Fig. 10 Canny operator

\section{Conclusions}

Edge detection is an important step in digital image processing. The paper introduced some operators of edge detection. Testing results shown that Rorberts operator detects edge accurately, but it is sensitive to noise; Prewitt operator can suppress noise; Sobel operator has a good performance in detecting edge; Canny operator makes good compromise between noise suppression and edge detection. The Sobel operator was the better one for iris identification systems due to better result, simplicity and easy to program.

\section{References}

[1] Kan Hao, Xu Guo-zhi. Iris Recognition System. Circuit and Systematic journal. 2000, 5(1), 11-15.

[2] T.Poggio, H.Voorhees and A.Yuille. "A Regularized Solution to Edge Detection". Tech. Rep. MA, Rep.AIM-833, MIT Artificial Intell. Lab. May, 1985.

[3] Zheng Nan-ning, Computer Vision and Pattern Recognition. Beijing. Publishing House of National Defense Industry, 1988.

[4] Zhang Yu-jin. Image Understanding and Image Analysis. Beijing. Publishing House of Tsinghua, 2000. 